\title{
ORTHOCOMPACTNESS IN INFINITE PRODUCT SPACES
}

\author{
NOBUYUKI KEMOTO AND YUKINOBU YAJIMA
}

(Communicated by Franklin D. Tall)

Dedicated to Professor Akihiro Okuyama on his 60th birthday

\begin{abstract}
In this paper, we prove the following results for an infinite product space $X=\prod_{\alpha \in \kappa} X_{\alpha}$.

(1) If a dense subspace of $X$ is orthocompact, then it is $\kappa$-metacompact.

(2) Assume that all finite subproducts of $X$ are hereditarily orthocompact. If a subspace of $X$ is $\kappa$-metacompact, then it is orthocompact.
\end{abstract}

\section{INTRODUCTION}

Throughout this paper, all spaces are assumed to be regular $T_{1}, \kappa$ denotes an infinite cardinal, and all product spaces are infinite product spaces. Whenever we consider a product space $\prod_{\alpha \in \kappa} X_{\alpha}$, we always assume that each $X_{\alpha}$ contains at least two points.

Beślagić [Be] proved that a product space $X=\prod_{\alpha \in \kappa} X_{\alpha}$ is $\kappa$-paracompact if it is normal. Conversely, Aoki [Ao] proved that a product space $X$ is normal (orthocompact) if it is $\kappa$-paracompact and each finite subproduct of $X$ is normal (orthocompact). In these connections, we recall Scott's result [S1] that a space $Z$ is $\kappa$-metacompact if $Z \times 2^{\kappa}$ is orthocompact.

In this paper, we prove that a dense subspace of a product space $X=\prod_{\alpha \in \kappa} X_{\alpha}$ is $\kappa$-metacompact if it is orthocompact. Conversely, we also prove that a product space $X$ is orthocompact if it is $\kappa$-metacompact and each finite subproduct of $X$ is hereditarily orthocompact. Moreover, we can give various applications of these results.

In the rest of this section, we state notation and basic facts. For a set $S$ and a cardinal $\lambda$, we define $[S]^{<\lambda}=\{T \subset S:|T|<\lambda\},[S]^{\leq \lambda}=\{T \subset S:|T| \leq \lambda\}$, and $[S]^{\lambda}=\{T \subset S:|T|=\lambda\}$, where $|T|$ denotes the cardinality of $T$. Let $\mathscr{U}$ be a collection of subsets of $S$ and $x \in S$. Then $(\mathscr{U})_{x}$ denotes $\{U \in \mathscr{U}: x \in$ $U$ \}. We say that a collection $\mathscr{V}$ of subsets of $S$ is a weak refinement of $\mathscr{U}$ if each member of $\mathscr{V}$ is contained in some member of $\mathscr{U}$. Furthermore, such a $\mathscr{V}$ is a refinement of $\mathscr{U}$ if $\bigcup \mathscr{V}=\bigcup \mathscr{U}$.

Received by the editors May 29, 1991.

1991 Mathematics Subject Classification. Primary 54B10, 54D20.

Key words and phrases. (Weakly) (sub)metacompact, (weakly) (sub)orthocompact, infinite product, $\Sigma$-product. 
Let $X=\prod_{\alpha \in \kappa} X_{\alpha}$ be a product space. For an $F \subset \kappa$, we denote by $X(F)$ the subproduct $\prod_{\alpha \in F} X_{\alpha}$ and denote by $\pi_{F}$ the canonical projection map $X \rightarrow$ $X(F)$. Such an $X(F)$ is called a finite subproduct of $X$ if $F$ is finite. In particular, we write $X_{\alpha}$ and $\pi_{\alpha}$ for $X(\{\alpha\})$ and $\pi_{\{\alpha\}}$, respectively.

A space $X$ is $(\kappa-)$ metacompact if each open cover of $X$ (with cardinality $\leq \kappa$ ) has a point-finite open refinement. A space $X$ is (weakly) submetacompact (or (weakly) $\theta$-refinable) if for each open cover $\mathscr{U}$ of $X$ there is a sequence $\left\{\mathscr{V}_{n}: n \in \omega\right\}$ of (weak) open refinements of $\mathscr{U}$ such that for each $x \in X$ there is an $n \in \omega$ such that $\left(x \in \bigcup \mathscr{V}_{n}\right.$ and) $\mathscr{V}_{n}$ is point-finite at $x$. We define (weak) $\kappa$-submetacompactness analogously. In particular, a sequence $\left\{\mathscr{V}_{n}: n \in \omega\right\}$ of covers of $X$ is called a $\theta$-sequence if for each $x \in X$ there is an $n \in \omega$ such that $\mathscr{V}_{n}$ is point-finite at $X$.

An open cover $\mathscr{V}$ of a space $X$ is interior-preserving if $\cap \mathscr{V}^{\prime}$ is open in $X$ for each $\mathscr{V}^{\prime} \subset \mathscr{V}$. A space $X$ is $(\kappa-)$ orthocompact if every open cover of $X$ (with cardinality $\leq \kappa$ ) has an interior-preserving open refinement. Note that a space $X$ is orthocompact if and only if every open cover $\mathscr{U}$ has an open refinement $\mathscr{V}$ of $\mathscr{U}$ such that $\bigcap(\mathscr{V})_{x}$ is a (an open) neighborhood of $x$. A space $X$ is (weakly) suborthocompact [KY, Ya] if for each open cover $\mathscr{U}$ of $X$ there is a sequence. $\left\{\mathscr{V}_{n}: n \in \omega\right\}$ of (weak) open refinements of $\mathscr{U}$ such that for each $x \in X$ there is an $n \in \omega$ such that $\bigcap\left(\mathscr{V}_{n}\right)_{x}$ is a neighborhood of $x$. We define (weak) $\kappa$-suborthocompactness analogously. In particular, a sequence $\left\{\mathscr{V}_{n}: n \in \omega\right\}$ of covers of $X$ is called an $l$-sequence [KY] if for each $x \in X$ there is an $n \in \omega$ such that $\bigcap\left(\mathscr{V}_{n}\right)_{x}$ is a neighborhood of $x$. Clearly, each $\theta$-sequence of open covers of $X$ is an $l$-sequence.

By these definitions, the following diagram is easily verified. But note that the ordinal space $\omega_{1}$ is (hereditarily) orthocompact but not weakly $\left(\omega_{1-}^{-}\right)$submetacompact.

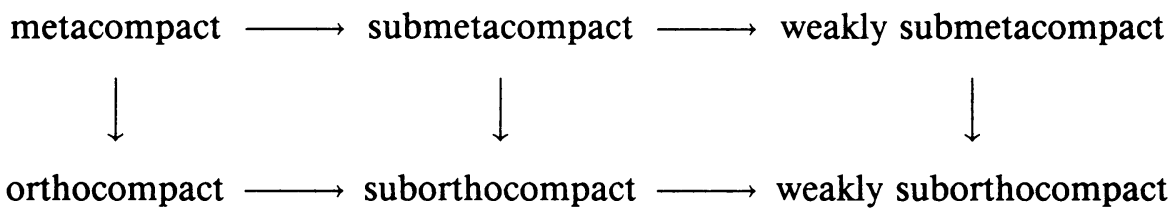

\section{2. $\kappa$-ORTHOCOMPACTNESS IN PRODUCT SPACES}

Theorem 2.1. Let $Y$ be a dense subspace of a product space $X=\prod_{\alpha \in \kappa} X_{\alpha}$. Then $Y$ is $\kappa$-metacompact ( $\kappa$-submetacompact, weakly $\kappa$-submetacompact) if and only if it is $\kappa$-orthocompact ( $\kappa$-suborthocompact, weakly $\kappa$-suborthocompact).

Proof. We prove the "if" part. Let $\mathscr{U}=\left\{U_{\alpha}: \alpha \in \kappa\right\}$ be an open cover of $Y$ with cardinality $\leq \kappa$. First we show that $\mathscr{U}$ has an open refinement (in $Y$ ) $\mathscr{W}$ of cardinality $\leq \kappa$ such that $\operatorname{int}_{Y}\left(\cap \mathscr{W}^{\prime}\right)=0$ for each $\mathscr{W}^{\prime} \in\left[\mathscr{W}^{\omega}\right.$.

For each $\alpha \in \kappa$, pick distinct two points $p_{\alpha}(0)$ and $p_{\alpha}(1)$ in $X_{\alpha}$. Since $X_{\alpha}$ is regular $T_{1}$, we take an open neighborhood $N_{\alpha}(i)$ of $p_{\alpha}(i)$, where $i \in$ $2=\{0,1\}$, such that $X_{\alpha}=N_{\alpha}(0) \cup N_{\alpha}(1)$ and $p_{\alpha}(1-i) \notin \operatorname{cl}_{X_{\alpha}} N_{\alpha}(i)$ for each $\alpha \in \kappa$ and each $i \in 2$. Let $G_{\alpha}(i)=\pi_{\alpha}^{-1}\left(N_{\alpha}(i)\right) \cap Y$ for each $\alpha \in \kappa$ and each $i \in 2$. Note that each $G_{\alpha}(i)$ is open in $Y$ and $Y=G_{\alpha}(0) \cup G_{\alpha}(1)$ for each $\alpha \in \kappa$.

Claim. $\operatorname{int}_{Y}\left(\bigcap_{\alpha \in A} G_{\alpha}(i)\right)=0$ for each $A \in[\kappa]^{\omega}$ and each $i \in 2$. 
Proof. Assume that there are an $A \in[\kappa]^{\omega}$, an $i \in 2$, and a $y \in Y$ such that $y \in \operatorname{int}_{Y}\left(\bigcap_{\alpha \in A} G_{\alpha}(i)\right)$. Then there are an $F \in[\kappa]^{<\omega}$ and an open set $V$ in $X(F)$ such that $y \in \pi_{F}^{-1}(V) \cap Y \subset \bigcap_{\alpha \in A} G_{\alpha}(i)$. Since $F$ is finite and $A$ is infinite, pick a $\beta$ in $A-F$. Since $Y$ is dense in $X$ and $\beta \notin F$, there is a point $z$ in $\pi_{F}^{-1}(V) \cap \pi_{\beta}^{-1}\left(X_{\beta}-\operatorname{cl} N_{\beta}(i)\right) \cap Y$. Then we have $z \in \pi_{F}^{-1}(V) \cap Y \subset$ $G_{\beta}(i) \subset \pi_{\beta}^{-1}\left(N_{\beta}(i)\right)$. This contradicts $z \in \pi_{\beta}^{-1}\left(X_{\beta}-\mathrm{cl} N_{\beta}(i)\right)$ and completes the proof of the claim.

Put $\mathscr{W}=\left\{U_{\alpha} \cap G_{\alpha}(i): \alpha \in \kappa\right.$ and $\left.i \in 2\right\}$. Then it follows from the claim that $\mathscr{W}$ is a desired open refinement of $\mathscr{U}$.

Now, we prove only the second case. From the above argument, we may assume that $\mathscr{U}=\left\{U_{\alpha}: \alpha \in \kappa\right\}$ is an open cover of $Y$ such that $\operatorname{int}_{Y}\left(\bigcap \mathscr{U}^{\prime}\right)=0$ for each $\mathscr{U}^{\prime} \in[\mathscr{U}]^{\omega}$. There is an $l$-sequence $\left\{\mathscr{V}_{n}: n \in \omega\right\}$ of open refinements of $\mathscr{U}$. Then it is easy to see that each $\mathscr{V}_{n}$ may be assumed to be a precise open refinement of $\mathscr{U}$, that is, $\mathscr{V}_{n}=\left\{V_{n}(U): U \in \mathscr{U}\right\}$ such that $V_{n}(U) \subset U$ for each $U \in \mathscr{U}$. Pick an $x \in Y$. Choose an $n \in \omega$ such that $\bigcap\left(\mathscr{V}_{n}\right)_{x}$ is a neighborhood of $x$. Assume that $\left(\mathscr{V}_{n}\right)_{x}$ is infinite. Then there is some $\mathscr{U}^{\prime} \in[\mathscr{U}]^{\omega}$ such that $\left\{V_{n}(U): U \in \mathscr{U}^{\prime}\right\} \subset\left(\mathscr{V}_{n}\right)_{x}$. So we have

$$
x \in \operatorname{int}_{Y}\left(\bigcap\left(\mathscr{V}_{n}\right)_{x}\right) \subset \operatorname{int}_{Y}\left(\bigcap\left\{V_{n}(U): U \in \mathscr{U}^{\prime}\right\}\right) \subset \operatorname{int}_{Y}\left(\bigcap \mathscr{U}^{\prime}\right)=0 .
$$

This is a contradiction. Thus, $\left\{\mathscr{V}_{n}: n \in \omega\right\}$ is a $\theta$-sequence of open refinements of $\mathscr{U}$. The proof is complete.

Corollary 2.2. If a product space $X=\prod_{\alpha \in \kappa} X_{\alpha}$ is orthocompact (suborthocompact, weakly suborthocompact), then $X$ is $\kappa$-metacompact ( $\kappa$-submetacompact, weakly $\kappa$-submetacompact).

For a space $X, L(X)$ denotes the Lindelöf degree of $X$.

Corollary 2.3 [S1, Ya]. A space $X$ is metacompact (submetacompact, weakly submetacompact) if and only if $X \times 2^{\kappa}$ is orthocompact (suborthocompact, weakly suborthocompact) where $L(X) \leq \kappa$.

Remark. Moreover, we can easily obtain the analogies of [Ao, Theorem 3.1]: A space $X$ is (weakly) $\kappa$-submetacompact if and only if $X \times A(\kappa)$ is (weakly) $\kappa$-suborthocompact, where $A(\kappa)$ is the one-point compactification of a discrete space of cardinality $\kappa$. Observe that this is a generalization of Corollary 2.3.

It is known that $\omega^{\omega_{1}}$ is not orthocompact; see [Ao, Theorem 3.4] or [S2, Theorem 2.4]. Moreover, we have

Corollary 2.4. $\omega^{\omega_{1}}$ is not suborthocompact.

Proof. Assume that $X=\omega^{\omega_{1}}$ is suborthocompact. Then, by Corollary 2.2, $X$ is $\omega_{1}$-submetacompact. Since the weight of $X$ is $\omega_{1}$, it is submetacompact. But it follows from the statement in [PP, p. 63] that $X$ is not submetacompact. This is a contradiction.

Let $Y$ be a $\Sigma$-product of $\left\{X_{\alpha}: \alpha \in \kappa\right\}$. Then $Y$ is said to be proper [Pr, $\S 7]$ if $Y$ is a proper subspace of $\prod_{\alpha \in \kappa} X_{\alpha}$ (i.e., $\kappa \geq \omega_{1}$ and $\left|X_{\alpha}\right| \geq 2$ for each $\alpha \in \kappa)$.

Corollary 2.5. All proper $\Sigma$-products are not weakly suborthocompact.

Proof. Let $Y$ be a proper $\Sigma$-product of $\left\{X_{\alpha}: \alpha \in \kappa\right\}$, where $\kappa \geq \omega_{1}$. Assume that $Y$ is weakly suborthocompact. Since $Y$ is dense in $X$, it follows from 
Theorem 2.1 that $Y$ is weakly $\kappa$-submetacompact. Since $Y$ contains a closed subspace which is homeomorphic to the ordinal space $\omega_{1}$ (cf. [Pr, Proposition 7.2]), the space $\omega_{1}$ is weakly $\kappa$-submetacompact. But it is well known that the space $\omega_{1}$ is not weakly $\omega_{1}$-submetacompact. This is a contradiction.

Corollary 2.6. Let $X$ be a product space of paracompact p-spaces (e.g., metrizable spaces). Then the following are equivalent.

(1) $X$ is (sub)orthocompact.

(2) $X$ is normal.

(3) $X$ is paracompact.

Using Corollary 2.4, the proof is similar to that of [ $\mathrm{Pr}$, Corollary 6.5].

Remark. The condition "paracompact $p$-space" in Corollary 2.6 is essential. In fact, let $X$ be a $\Sigma$-product in $2^{\omega_{1}}$. Then $X$ is homeomorphic to $X^{\omega}$. It follows from [Pr, Theorem 7.4] and Corollary 2.5 that $X^{\omega}$ is normal but not weakly suborthocompact.

We obtain the following generalization of [Ao, Theorem 3.5] or [S2, Theorem 2.5].

Corollary 2.7. The following are equivalent for a space $X$.

(1) $X$ is compact.

(2) $X^{\kappa}$ is suborthocompact for any cardinal $\kappa$.

(3) $X^{\kappa}$ is suborthocompact for some cardinal $\kappa$ with $\kappa \geq \omega_{1} \cdot L(X)$. 3.5].

Using Corollaries 2.3 and 2.4, the proof is parallel to that of [Ao, Theorem

If $\omega^{\omega_{1}}$ was not weakly submetacompact, then "suborthocompact" in most of our corollaries could be replaced by "weakly suborthocompact". Hence, we conclude this section with the following problem.

Problem 2.8. Is $\omega^{\omega_{1}}$ not weakly submetacompact?

\section{3. $\kappa$-METACOMPACTNESS IN PRODUCT SPACES}

As the converse of Corollary 2.2, we obtain the following:

Theorem 3.1. Assume that all finite subproducts of a product space $X=\prod_{\alpha \in \kappa} X_{\alpha}$ are hereditarily orthocompact. If a subspace $Y$ of $X$ is $\kappa$-metacompact $(\kappa$ submetacompact, weakly $\kappa$-submetacompact), then it is orthocompact (suborthocompact, weakly suborthocompact).

Proof. We prove only the second case. Let $\mathscr{U}$ be an open cover of $Y$. We may assume that, for each $U \in \mathscr{U}$, there are an $F(U) \in[\kappa]^{<\omega}$ and an open set $G(U)$ in $X(F(U))$ such that $U=\pi_{F(U)}^{-1}(G(U)) \cap Y$. For each $F \in[\kappa]^{<\omega}$, put $\mathscr{U}_{F}=\{U \in \mathscr{U}: F(U)=F\}$ and $G_{F}=\bigcup\left\{G(U): U \in \mathscr{U}_{F}\right\}$. Then it is easy to check that each $G_{F}$ is open in $X(F)$ and $\mathscr{A}=\left\{\pi_{F}^{-1}\left(G_{F}\right) \cap Y: F \in[\kappa]^{<\omega}\right\}$ is an open cover of $Y$. Since each $X(F)$ is hereditarily orthocompact, there is an interior-preserving collection $\mathscr{B}(F)=\left\{B_{F}(U): U \in \mathscr{U}_{F}\right\}$ of open sets in $X(F)$ such that $B_{F}(U) \subset G(U)$ for each $U \in \mathscr{U}_{F}$ and $\bigcup \mathscr{B}(F)=G_{F}$. By the $\kappa$-submetacompactness of $Y$ and $|\mathscr{A}| \leq \kappa$, there is a $\theta$-sequence $\left\{\mathscr{V}_{n}: n \in \omega\right\}$ of open refinements of $\mathscr{A}$. We may assume that $\mathscr{V}_{n}=\left\{V_{F}^{n}: F \in[\kappa]^{<\omega}\right\}$ 
such that $V_{F}^{n} \subset \pi_{F}^{-1}\left(G_{F}\right) \cap Y$ for each $F \in[\kappa]^{<\omega}$ and each $n \in \omega$. Put $\mathscr{W}_{F}^{n}=\left\{\pi_{F}^{-1}\left(B_{F}(U)\right) \cap V_{F}^{n}: U \in \mathscr{U}_{F}\right\}$ for each $F \in[\kappa]^{<\omega}$ and each $n \in \omega$. Then it is easy to check that each $\mathscr{W}_{F}^{n}$ is an interior-preserving collection of open sets in $Y$ whose union is $V_{F}^{n}$. Put $\mathscr{W}_{n}=\bigcup\left\{\mathscr{W}_{F}^{n}: F \in[\kappa]^{<\omega}\right\}$ for each $n \in \omega$. Observe that each $\mathscr{W}_{n}$ is an open refinement of $\mathscr{U}$. We show that $\left\{\mathscr{W}_{n}: n \in \omega\right\}$ is an $l$-sequence. Pick an $x \in X$. Since $\left\{\mathscr{V}_{n}: n \in \omega\right\}$ is a $\theta$-sequence, take an $n \in \omega$ such that $\left(\mathscr{V}_{n}\right)_{x}$ is finite, say $\left(\mathscr{V}_{n}\right)_{x}=\left\{V_{F}^{n}: F \in \mathscr{F}\right\}$ for some $\mathscr{F} \in$ $\left[[\kappa]^{<\omega}\right]^{<\omega}$. Since $\mathscr{W}_{F}^{n}$ is interior-preserving and $x \in V_{F}^{n}=\bigcup \mathscr{W}_{F}^{n}, \bigcap\left(\mathscr{W}_{F}^{n}\right)_{x}$ is an open neighborhood of $x$ for each $F \in \mathscr{F}$. Since $\left(\mathscr{W}_{n}\right)_{x}=\bigcup_{F \in \mathscr{F}}\left(\mathscr{W}_{F}^{n}\right)_{x}$ and $|\mathscr{F}|<\omega$, it follows that $\bigcap\left(\mathscr{W}_{n}\right)_{x}=\bigcap_{F \in \mathscr{F}}\left(\bigcap\left(\mathscr{W}_{F}^{n}\right)_{x}\right)$ is an open neighborhood of $x$. This completes the proof.

Considering [Ao, Corollary 2.5], it is natural to raise

Problem 3.2. If a product space $X=\prod_{\alpha \in \kappa} X_{\alpha}$ is $\kappa$-metacompact and all finite subproducts of $X$ are orthocompact, is $X$ orthocompact?

Proposition 3.3. Assume that all finite subproducts of a product space $X=$ $\prod_{\alpha \in \kappa} X_{\alpha}$ are hereditarily metacompact. If a dense subspace $Y$ of $X$ is $\kappa$ orthocompact ( $\kappa$-suborthocompact, weakly $\kappa$-suborthocompact), then $Y$ is metacompact (submetacompact, weakly submetacompact).

Proof. The second case: Observe that $Y$ is $\kappa$-submetacompact according to Theorem 2.1 , because $Y$ is a $\kappa$-suborthocompact dense subspace of $X$. Then replacing "interior-preserving" by "point-finite" in the proof of Theorem 3.1, we can prove similarly.

Corollary 3.4. Let $X$ be a product space of metrizable spaces and $Y$ a dense subspace of $X$. Then $Y$ is orthocompact (suborthocompact, weakly suborthocompact) if and only if it is metacompact (submetacompact, weakly submetacompact).

We can consider this is an analogue of [ $\mathrm{Ba}$, Theorem 1].

Remark. Under the assumption of Corollary 3.4, $X$ is normal if and only if it is paracompact (see Corollary 2.6). But one cannot replace "orthocompact" and "metacompact" by "normal" and "paracompact", respectively, in Corollary 3.4. In fact, let $Y$ be a $\Sigma$-product in $X=2^{\omega_{1}}$. Then $Y$ is a normal nonparacompact, dense subspace of $X$.

\section{REFERENCES}

[Ao] Y. Aoki, Orthocompactness of inverse limits and products, Tsukuba J. Math. 4 (1980), 241255.

[Ba] D. P. Baturov, Normality in dense subspaces of products, Topology Appl. 36 (1990), 111-116.

[Be] A. Beślagić, Normality in products, Topology Appl. 22 (1986), 71-82.

[KY] N. Kemoto and Y. Yajima, Orthocompactness in products, Tsukuba J. Math. 16 (1992), 407-422.

[PP] R. Pol and E. Puzio-Pol, Remarks on Cartesian products, Fund. Math. 93 (1976), 57-69.

[Pr] T. C. Przymusiński, Products of normal spaces, Handbook of Set Theoretic Topology (K. Kunen and J. E. Vaughan, eds.), North-Holland, Amsterdam, 1984, pp. 781-826.

[S1] B. M. Scott, Toward a product theory for orthocompactness, Studies in Topology (N. M. Stavrakas and K. R. Allen, eds.), Academic Press, New York, 1975, pp. 517-537. 
[S2] - More about orthocompactness, Topology Proc. 5 (1980), 155-184.

[Ya] Y. Yajima, A characterization of submetacompactness in terms of products, Proc. Amer. Math. Soc. 112 (1991), 291-296.

Department of Mathematics, Faculty of Education, Oita University, Dannoharu, OITA, 870-11 JAPAN

E-mail address: nkemoto৫cc.oita-u.ac.jp

Department of Mathematics, Kanagawa University, Yokohama, 221 Japan

E-mail address: yuki@kani.cc.kanagawa-u.ac.jp 